\title{
An experimental comparison of evaporation and condensation heat transfer coefficients for HFC-134a and CFC-12
}

\author{
S. J. Eckels and M. B. Pate \\ Department of Mechanical Engineering, lowa State University, Ames, IA 50011, USA
}

Experimental heat transfer coefficients are reported for HFC-134a and CFC-12 during in-tube single-phase flow, evaporation and condensation. These heat transfer coefficients were measured in a horizontal, smooth tube with an inner diameter of $8.0 \mathrm{~mm}$ and a length of $3.67 \mathrm{~m}$. The refrigerant in the test-tube was heated or cooled by using water flowing through an annulus surrounding the tube. Evaporation tests were performed for a refrigerant temperature range of $5-15^{\circ} \mathrm{C}$ with inlet and exit qualities of 10 and $90 \%$, respectively. For condensation tests, the refrigerant temperature ranged from 30 to $50^{\circ} \mathrm{C}$, with inlet and exit qualities of 90 and $10 \%$, respectively. The mass flux was varied from 125 to $400 \mathrm{~kg} /\left(\mathrm{m}^{2} \mathrm{~s}\right)$ for all tests. For similar mass fluxes, the evaporation and condensation heat transfer coefficients for HFC-134a were significantly higher than those of CFC-12. Specifically, HFC-134a showed a $35-45 \%$ increase over CFC-12 for evaporation and a $25-35 \%$ increase over CFC- 12 for condensation.

\section{Nomenclature}

\begin{tabular}{|c|c|c|}
\hline & $x$ & Quality (\%) \\
\hline \multicolumn{2}{|l|}{$A$} & Area $\left(m^{2}\right)$ \\
\hline \multicolumn{2}{|l|}{ C } & Specific heat $(k J /(k g K))$ \\
\hline \multicolumn{2}{|l|}{$h$} & Convective heat transfer coefficient $\left(W /\left(m^{2} K\right)\right)$ \\
\hline \multicolumn{2}{|l|}{ I } & Current $(A)$ \\
\hline \multicolumn{2}{|l|}{$\mathrm{I}_{\mathrm{fg}}$} & Enthalpy of vaporization $(\mathrm{kJ} / \mathrm{kg})$ \\
\hline \multicolumn{2}{|l|}{ LMTD } & Log mean temperature difference \\
\hline \multicolumn{2}{|l|}{$\mathrm{m}$} & Mass flowrate $(\mathrm{kg} / \mathrm{s})$ \\
\hline \multicolumn{2}{|l|}{ Q } & Heat transfer rate $\left(W /\left(m^{2} K\right)\right)$ \\
\hline \multicolumn{2}{|l|}{$\mathrm{T}$} & Temperature $\left({ }^{\circ} \mathrm{C}\right)$ \\
\hline \multicolumn{2}{|l|}{ U } & Overall heat transfer coefficient $\left(W /\left(m^{2} K\right)\right)$ \\
\hline \multicolumn{2}{|l|}{ v } & Voltage (V) \\
\hline \multicolumn{3}{|c|}{ Subscripts } \\
\hline $\mathrm{BL}$ & \multicolumn{2}{|c|}{ Boiler } \\
\hline 1 & \multicolumn{2}{|c|}{ Inner tube } \\
\hline 10 & \multicolumn{2}{|c|}{ Inlet } \\
\hline $\mathrm{H}$ & \multicolumn{2}{|l|}{ Heater } \\
\hline 0 & \multicolumn{2}{|l|}{ Annulus } \\
\hline out & \multicolumn{2}{|l|}{ Outlet } \\
\hline $\mathrm{R}$ & \multicolumn{2}{|c|}{ Refrigerant } \\
\hline sat & \multicolumn{2}{|c|}{ Saturation } \\
\hline $\mathrm{T}$ & \multicolumn{2}{|c|}{ Test section } \\
\hline $\mathrm{SH}$ & \multicolumn{2}{|c|}{ Superheater } \\
\hline
\end{tabular}


Eckels, S.J. and M.B. Pate, An experimental comparison of evaporation and condensation heat transfer coefficients for HFC-134a and CFC-12. International Journal of Refrigeration, 1991. 14(2): p. 70-77.

W Water

\section{Introduction}

Experimental heat transfer evaluations of HFC-134a and other alternative refrigerants have become increasingly important as reductions in CFCs take effect. As the thermodynamic properties of the two refrigerants are similar, HFC-134a is considered a potential replacement for CFC-12. HFC-134a is also more environmentally acceptable with a zero-ozone depletion factor.

The goal of this research was to determine evaporation and condensation heat transfer coefficients of HFC134a. Measurements for CFC-12 were also taken to provide a base line for judging the performance of HFC134a. A lack of published information on the heat transfer characteristics of HFC-134a prevented any comparisons with other experimental work. However, it was possible to compare the experimental results for HFC-134a with predicted heat transfer coefficients obtained from theoretical correlations. Heat transfer coefficients for HFC-134a and CFC-12 were determined with an experimental rig capable of testing both singlephase and two-phase flow. This paper will review the main aspects of this experimental rig and the operational procedures used during testing. The main equations used in the data reduction are also reviewed. Results for single-phase evaporation and condensation of both HFC-134a and CFC-12 are given and the performance of the two refrigerants are compared.

\section{Test facilities}

The test rig has four main parts: a refrigerant loop, a water loop, a water-glycol loop and a data acquisition system. The refrigerant loop contains the test section, which consists of a horizontal smooth tube. The water loop contains the annulus that surrounds the test section and is used to heat or cool the refrigerant during testing. The water-glycol loop is used to subcool the refrigerant that leaves the test section. All system data taken during a test run are recorded by the data acquisition system. A schematic diagram of the test rig is shown in Figure 1

\section{Refrigerant loop}

The refrigerant loop contains the test section, an after-condenser, a positive displacement pump, an accumulator bladder, a boiler and superheater. The test section is a horizontal smooth tube surrounded by an annulus. The inner tube in which the refrigerant flows is a $3.67 \mathrm{~m}$ long smooth tube with an outer diameter of $9.25 \mathrm{~mm}$ and an inner diameter of $8.0 \mathrm{~mm}$. The refrigerant is heated or cooled during testing by the water flowing in the outer annulus.

The after-condenser is a coaxial heat exchanger that subcools the refrigerant exiting the test section to $-15^{\circ} \mathrm{C}$. The subcooled refrigerant is then pumped with a positive displacement pump, which allows oil-free circulation in the refrigerant loop. The flowrate through the test section is regulated by valves and a bypass line. The accumulator bladder sets the system pressure and dampens any fluctuations in the system. With the flowrate and pressure set, the quality entering the test section is controlled by using two heaters, namely a boiler and superheater, located just before the test section. The boiler is a $12.7 \mathrm{~mm}$ o.d., $2.63 \mathrm{~m}$ long stainless-steel tube heated by direct electric current, whereas the superheater is a $12.7 \mathrm{~mm}$ o.d., $1.83 \mathrm{~m}$ long copper tube wrapped with a ceramic bead heater supplied with alternating current.

The refrigerant flow rate is measured by a positive displacement flow meter with an experimental uncertainty of $\pm 1 \%$. The refrigerant pressure is measured at the inlet of the test section with a strain gauge type pressure transducer, accurate to $\pm 9 \mathrm{kPa}$. The pressure drop of the refrigerant flowing through the test section is measured with a strain-gauge type differential pressure transducer accurate to $\pm 0.2 \mathrm{kPa}$. A pair of thermocouples is located at the inlet and another pair at the exit of the test section. 


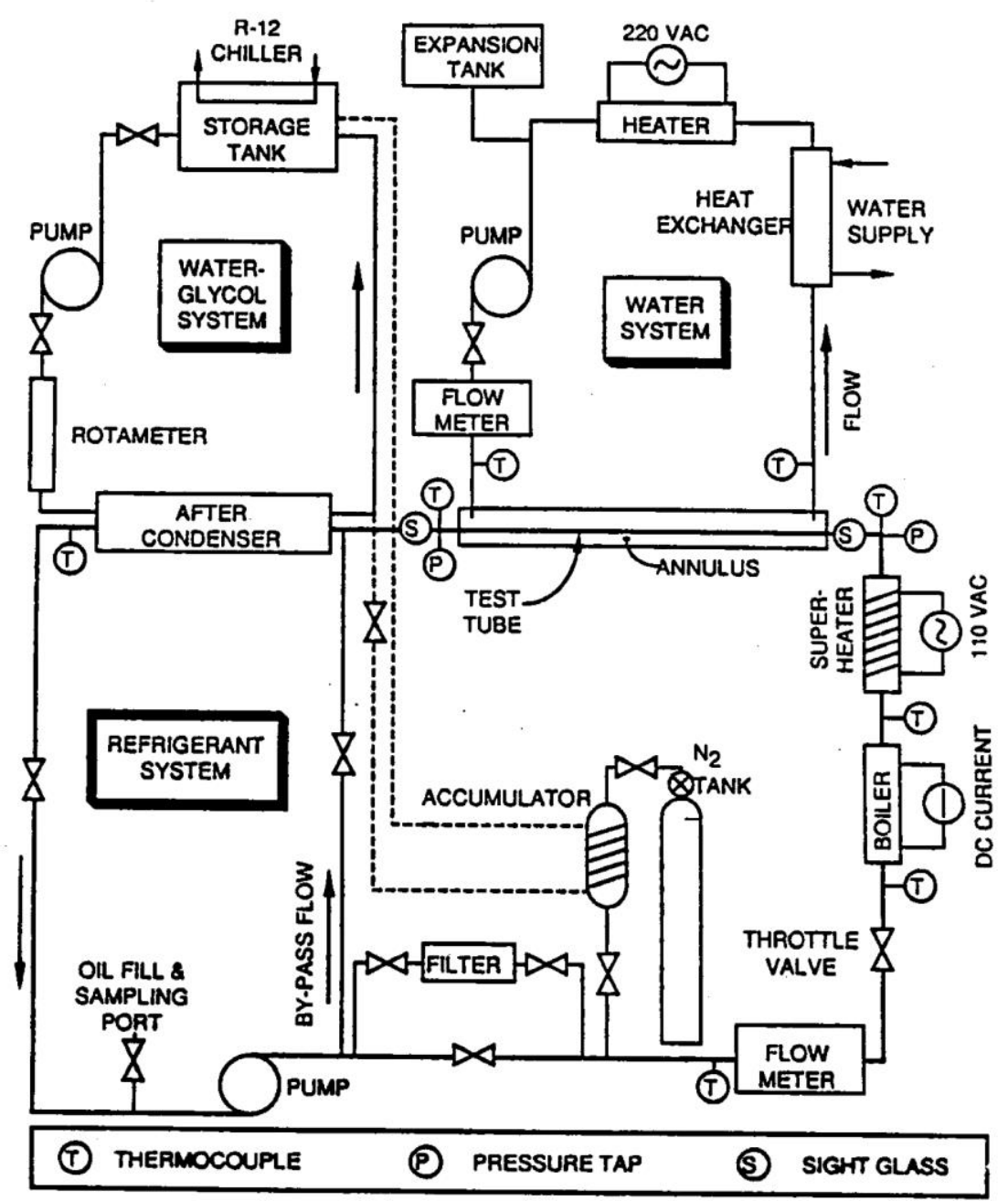

Figure 1: Schematic diagram of test facilities

\section{Water loop}

The water loop is used to supply water to the annulus side of the test section for the purpose of heating or cooling the refrigerant flowing in the test-tube. The water loop consists of the test section annulus, a centrifugal pump, a magnetic flow meter, and a heat exchanger. The water flowrate is set by the centrifugal pump and a restricting valve. The temperature of the water in the line is controlled by the heat exchanger which can be supplied with warm or cold water from the building taps, depending on the temperature that is needed in the annulus. The magnetic flow meter measures the water flowrate with an experimental uncertainty of $\pm 2 \%$. The temperature in the annulus is measured by two pairs of thermocouples with one pair located at the inlet and the other pair at the exit of the annulus. From calibration of the thermocouples, an uncertainty of $\pm 0.2^{\circ} \mathrm{C}$ was found for the temperature difference measurements.

\section{Water-glycol loop}

The water-glycol loop contains a storage tank with a 209 L capacity, a centrifugal pump, a coaxial heat exchanger, and a $17.5 \mathrm{~kW}$ refrigeration unit. The refrigeration unit cools the fluid in the storage tank down to $-20^{\circ} \mathrm{C}$. To keep this fluid from freezing, a 50/50 mixture of water and glycol is used. This mixture is circulated by a centrifugal 
Eckels, S.J. and M.B. Pate, An experimental comparison of evaporation and condensation heat transfer coefficients for HFC-134a and CFC-12. International Journal of Refrigeration, 1991. 14(2): p. 70-77.

pump through the coaxial heat exchanger (in the refrigerant line) which is used to condense the refrigerant leaving the test section.

\section{Data acquisition}

The data acquisition system consists of a controller, a 40-channel scanner, and a multimeter. A FORTRAN program is used as the source code for the controller. The controller, scanner and multimeter communicate via an IEEE488 bus. The system automatically monitors 20 copper-constantan thermocouples, two pressure transducers, two flow meters and the voltage and current to the boiler. The ambient temperature and pressure are the only parameters that are entered manually.

\section{Experimental procedures}

The system is allowed to come to a steady state before final data acquisition is started. Achieving steady state involves setting the refrigerant flowrate, annulus temperature difference, refrigerant pressure and inlet and exit qualities of the refrigerant. A program in the controller checks for steady state by monitoring temperature changes in the system. When no changes in the temperature or flowrate can be detected, the final data acquisition program is initiated. This runs for $2 \mathrm{~min}$, scanning all channels a total of five times. Because of pressure fluctuations, the pressure drop channel is scanned a total of 35 times. The data for each channel is averaged and an arithmetic mean is calculated. If any large deviations due to unsteady effects are detected the run is aborted.

\section{Data reduction}

The main equations used in the data reduction are based on energy balances. The heat transferred in the test section is calculated from an energy balance on the water flowing in the annulus:

$$
Q_{T w}=M_{w} C_{w}\left(T_{W_{\text {in }}}-T_{W_{\text {out }}}\right)
$$

During single-phase flow, the heat transferred in the test section can also be calculated from an energy balance on the refrigerant:

$$
Q_{T_{R}}=M_{R} C_{R}\left(T_{R_{\text {in }}}-T_{R_{\text {out }}}\right)
$$

A comparison of the water-side energy balance and the refrigerant-side energy balance provides a relative check of measurement accuracy. These two energy balances agreed within $6 \%$ for all runs.

The vapor quality entering the test section can be calculated from an energy balance on the boiler and superheater. The heat output from the boiler and superheater can be calculated from current and voltage readings as follows:

$$
Q_{H}=H L\left[(V I)_{B L}+(V I)_{S H}\right]
$$

where the $\mathrm{HL}$ factor represents the heat lost to the environment. An energy balance on the heaters during singlephase flow resulted in a HL factor of 0.98 . The heat input to the refrigerant in the heaters takes two forms, sensible and latent:

$$
Q_{H}=Q_{\text {sens }}+Q_{\text {lat }}
$$

where:

$$
\begin{gathered}
Q_{\text {sens }}=M_{R} C_{R}\left(T_{\text {sat }}-T_{H_{\text {in }}}\right) \\
Q_{\text {lat }}=M_{R} i_{\text {fg }} X_{H_{\text {out }}}
\end{gathered}
$$

The saturation temperature of the refrigerant is determined from the saturation pressure at the inlet of the test section. Equation (6) can then be used to determine the refrigerant quality exiting the heaters, which is the 
Eckels, S.J. and M.B. Pate, An experimental comparison of evaporation and condensation heat transfer coefficients for HFC-134a and CFC-12. International Journal of Refrigeration, 1991. 14(2): p. 70-77.

quality entering the test section. The quality change in the test section is calculated from the heat transferred in the test section and the refrigerant mass flowrate:

$$
\Delta X=\frac{Q_{T_{w}}}{M_{R} i_{f g}}
$$

Equations (6) and (7) are sufficient to determine the qualities in the test section as long as the superheated vapor region is not entered. For this study, vapor qualities were maintained at or below $90 \%$. The refrigerantside heat transfer coefficient can be determined from the overall heat transfer coefficient and the annulus-side heat transfer coefficient by using the procedures described below. The overall heat transfer coefficient is:

$$
U_{o}=\frac{Q_{T_{w}}}{A_{o} L M T D}
$$

The log mean temperature difference (LMTD) is determined from the annulus inlet and exit temperatures and from the saturation temperatures at the inlet and exit of the test section:

$$
\operatorname{LMTD}=\frac{\left(\Delta T_{1}-\Delta T_{2}\right)}{\ln \left(\Delta T_{1} / \Delta T_{2}\right)}
$$

where:

$$
\begin{aligned}
& \Delta T_{1}=T_{\text {sat }_{\text {out }}}-T_{W_{\text {in }}} \\
& \Delta T_{1}=T_{\text {sat }_{\text {in }}}-T_{W_{\text {out }}}
\end{aligned}
$$

The heat transfer coefficient of the water on the annulus side of the test section was calibrated by using a Wilson plot technique.': By assuming that the copper tube and fouling effects are negligible, the refrigerant heat transfer coefficient can be determined from:

$$
h_{i}=\frac{1}{\left(\frac{1}{U_{o}}-\frac{1}{h_{o}}\right) \frac{A_{i}}{A_{o}}}
$$

This equation determines an average heat transfer coefficient over the length of the tube. Both single-phase flow and two-phase use Equations (8)-(12) for calculating refrigerant-side heat transfer coefficients. However, if single-phase flow is present, then several adjustments are appropriate. Specifically, the LMTD of Equation (9) is calculated from the thermocouple reading at the inlet and exit of the test section and the $Q_{\text {Tw }}$ of Equation (8) is an average of the water-side and refrigerant-side energy balances.

A propagation or error method suggested by Kline and McCiintock[2] was used to estimate the experimental uncertainty. During evaporation of HFC-134a, the experimental uncertainty in the heat transfer coefficient was $\pm 9 \%$ at a mass flux of $400 \mathrm{~kg} /\left(\mathrm{m}^{2} \mathrm{~s}\right)^{1}$ and $\pm 14 \%$ at a mass flux of $130 \mathrm{~kg} /\left(\mathrm{m}^{2} \mathrm{~s}\right)$. For the condensation of HFC134a, the experimental uncertainty in the heat transfer coefficient was $\pm 8 \%$ at a mass flux of $400 \mathrm{~kg} /\left(\mathrm{m}^{2} \mathrm{~s}\right)$ and $\pm 13 \%$ at a mass flux of $130 \mathrm{~kg} /\left(\mathrm{m}^{2} \mathrm{~s}\right)$. The experimental uncertainties in the heat transfer coefficient for CFC-12 were similar to those for HFC-134a. The experimental uncertainty in the refrigerant mass flux was $\pm 3 \%$ whereas the quality had an experimental uncertainty of $\pm 3.5 \%$.

\section{Test results}

Experimental heat transfer coefficients are reported for HFC-134a and CFC-12 during condensation, evaporation and single-phase flow. As mentioned earlier, the test tube was a $3.67 \mathrm{~m}$ long smooth tube with an inner diameter of $8.0 \mathrm{~mm}$. The range of test conditions were selected to reflect actual conditions in refrigeration systems. As HFC$134 \mathrm{a}$ is a possible replacement for CFC-12, it is desirable to compare the refrigerants under the same operating 
Eckels, S.J. and M.B. Pate, An experimental comparison of evaporation and condensation heat transfer coefficients for HFC-134a and CFC-12. International Journal of Refrigeration, 1991. 14(2): p. 70-77.

conditions. The experimentally determined heat transfer coefficients are also used to determine which theoretical correlation best estimates the heat transfer coefficients for each refrigerant.

Single-phase flow

Liquid single-phase heat transfer coefficients were determined at average temperatures ranging from 24 to $27^{\circ} \mathrm{C}$ and at mass fluxes ranging from 500 to $900 \mathrm{~kg} /\left(\mathrm{m}^{2} \mathrm{~s}\right)$. Single-phase heat transfer tests were conducted because both evaporators and condensers operate with single-phase regions. Single-phase tests are also used as a check on system accuracy as single-phase heat transfer correlations from the literature can accurately predict heat transfer coefficients.

Figure 2 shows the liquid single-phase heat transfer coefficients for HFC-134a and CFC-12. The lines on the graph represent a least-squares fit of the plotted points for each refrigerant. For this series of tests, the refrigerant is being cooled. When the heat transfer coefficients for the two refrigerants are compared, HFC-134a shows a $30 \%$ increase over CFC-12. Most of the increase in heat transfer coefficients can be attributed to the increased liquid thermal conductivity of HFC-134a. For example, at $27^{\circ} \mathrm{C}$ the liquid thermal conductivity of $\mathrm{HFC}-134 \mathrm{a}$ is $81.4 \mathrm{~mW}$ $\mathrm{m}^{-1} \mathrm{~K}^{-1}$ while for CFC-12 it is $69.7 \mathrm{~mW} \mathrm{~m}^{-1} \mathrm{~K}^{-1}$ (ref. 3). This is a $17 \%$ increase in liquid thermal conductivity for HFC134a.

The experimentally determined Nusselt numbers for HFC-134a and CFC-12 are compared with the Dittus-BoelterMcadams [4] and Petukhov-Popov[5] correlations. Figure 3 shows the predicted Nusselt number versus the experimentally determined Nusselt number for HFC-134a and CFC-12. For HFC-134a the Petukhov-Popov correlation predicts the experimental Nusselt number within $2.5 \%$, whereas the Dittus-Boelter predicts Nusselt number within $24 \%$. For CFC-12 the Petukhov- Popov correlation predicts the experimental Nusselt number within $1.5 \%$, whereas the Dittus-Boelter correlation predicts Nusselt number within $25 \%$. The Petukhov-Popov has also been shown to be accurate for other refrigerants, such as HCFC-22 [1].

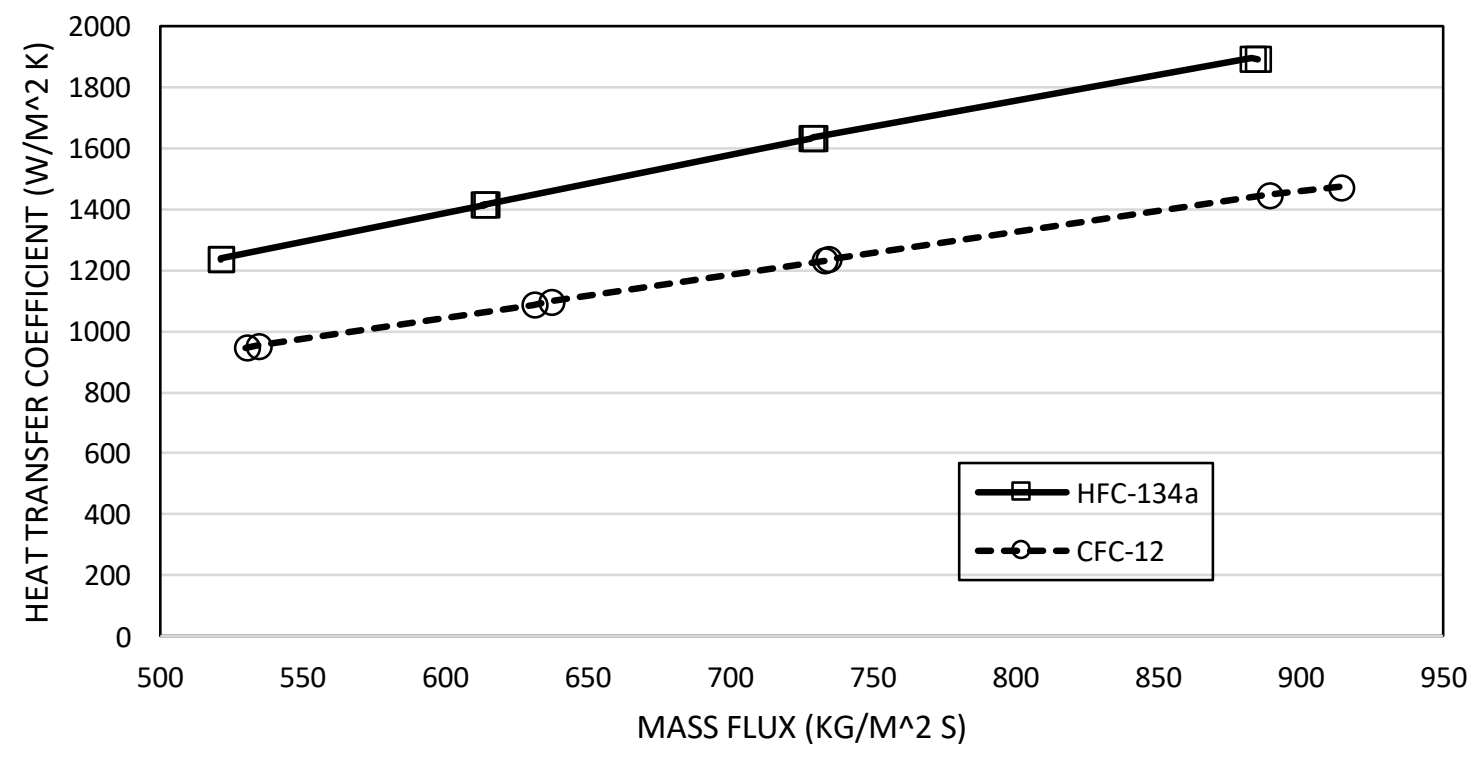

Figure 2 Measured single-phase heat transfer coefficients for HFC-134a and CFC-12 
Eckels, S.J. and M.B. Pate, An experimental comparison of evaporation and condensation heat transfer coefficients for HFC-134a and CFC-12. International Journal of Refrigeration, 1991. 14(2): p. 70-77.

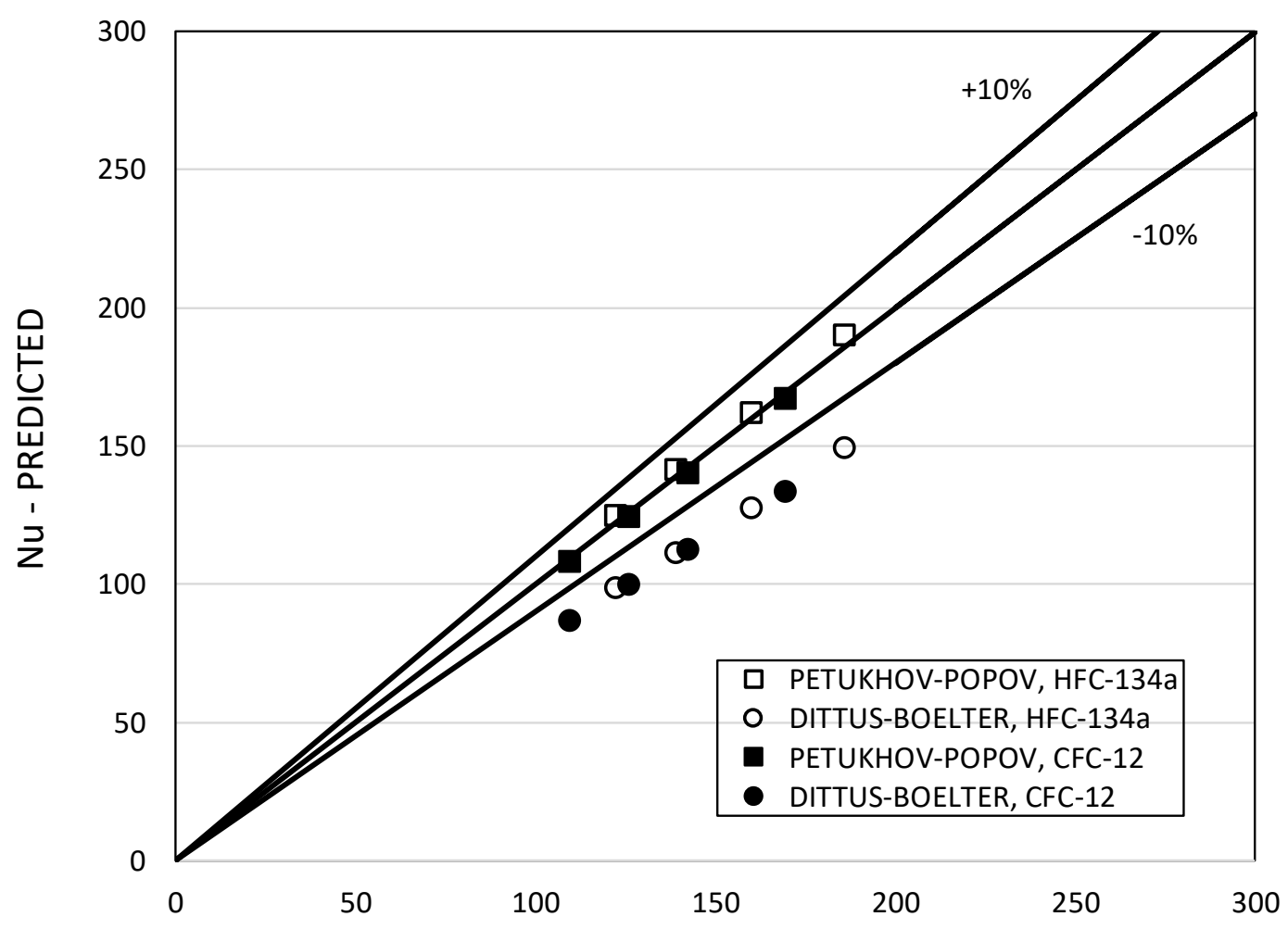

$\mathrm{NU}$ - EXPERIMENTAL

Figure 3: Predicted Nusselt number for CFC-12 and HFC-134a versus experimentally determined Nusselt number. (squares) Petukhov-Popov; (circles) Dittus-Boelter CFC-12

Table 1 Evaporation test conditions

\begin{tabular}{|l|l|l|}
\hline Parameter & CFC-12 & HFC-134a \\
\hline Temperature $\left({ }^{\circ} \mathrm{C}\right)$ & $5-15$ & $5-15$ \\
\hline Pressure $(\mathrm{MPa})$ & $0.36-0.49$ & $0.35-0.49$ \\
\hline Mass flux $\left(\mathrm{kg} /\left(\mathrm{m}^{2} \mathrm{~s}\right)\right)$ & $125-400$ & $125-400$ \\
\hline Quality in $(\%)$ & $5-15$ & $5-15$ \\
\hline Quality out $(\%)$ & $80-88$ & $80-88$ \\
\hline
\end{tabular}

\section{Evaporation}

Evaporation tests were performed over a range of mass fluxes at three temperatures: 5,10 and $15^{\circ} \mathrm{C}$. Although attempts were made to match the above temperatures, slight variations in evaporation temperature, $\pm 1^{\circ} \mathrm{C}$, occurred between tests. The conditions for evaporation tests are summarized in Table 1 . When the mass fluxes are fixed for the two refrigerants, the heat flux must be varied to obtain similar exiting qualities for the two refrigerants. This reflects the increased enthalpy of vaporization for HFC-134a. For example, at a mass flux of 200 $\mathrm{kg} /\left(\mathrm{m}^{2} \mathrm{~s}\right)$ and a temperature of $10^{\circ} \mathrm{C}$, the heat flux was $12.1 \mathrm{~kW} / \mathrm{m}^{2}$ for HFC-134a and $9.1 \mathrm{~kW} / \mathrm{m}^{2}$ for CFC12. 
Eckels, S.J. and M.B. Pate, An experimental comparison of evaporation and condensation heat transfer coefficients for HFC-134a and CFC-12. International Journal of Refrigeration, 1991. 14(2): p. 70-77.

Figure 4 shows evaporation heat transfer coefficient data versus mass flux for HFC-134a and CFC-12. The line representing each temperature is a least-squares fit of the data obtained at that temperature. Both HFC-134a and CFC-12 heat transfer coefficients increased with temperature and mass flux. Of special importance, HFC-134a shows a significant increase in evaporation heat transfer coefficients compared to CFC-12, with increases ranging from 30 to $40 \%$. Part of the increase in the evaporation heat transfer coefficients for HFC-134a is due to the higher heat flux mentioned earlier. The relationship between increased heat flux and the increase in the HFC-134a heat transfer coefficients was estimated using well known correlations. The correlations predicted that the higher heat flux for HFC-134a increases the evaporation heat transfer coefficient by as much as $10 \%$ over the values for CFC12. However, the increase for HFC-134a is significantly higher than this value. A more detailed comparison of the two refrigerants is presented in a later section.

The experimental data are compared to predictions from the correlations of Shah[6], Kandlikar[7], Chaddock and Brunemann[8], and Gungor and Winterton[9]. Local heat transfer coefficients from these correlations were numerically integrated over the whole quality range to obtain average heat transfer coefficients. Figure 5 shows the CFC-12 heat transfer coefficients versus the predicted heat transfer coefficients. For CFC-12, all correlations, except the Chaddock-Brunernann[8] correlation, differ from experimental results by less than $\pm 25 \%$. The differences for the Shah[6] correlation are even smaller, being less than $\pm 15 \%$. Figure 6 compares experimental heat transfer coefficients for HFC-134a with the theoretical correlations. The differences for all correlations are less than $\pm 25 \%$, with the Chaddock-Brunemann[8] and Kandlikar[7] correlation results differing from experimental results by less than $\pm 15 \%$. It should be noted that for HFC-134a a fluid dependent factor equivalent to the CFC-12 value, namely 1.5, was used in the Kandlikar correlation shown in Figure 6. However, the experimental data for HFC-134a was also used to determine the fluid dependent factor in the Kandlikar correlation. A value of 1.63 for the fluid dependent factor was found to give the lowest deviation for all HFC-134a experimental data.

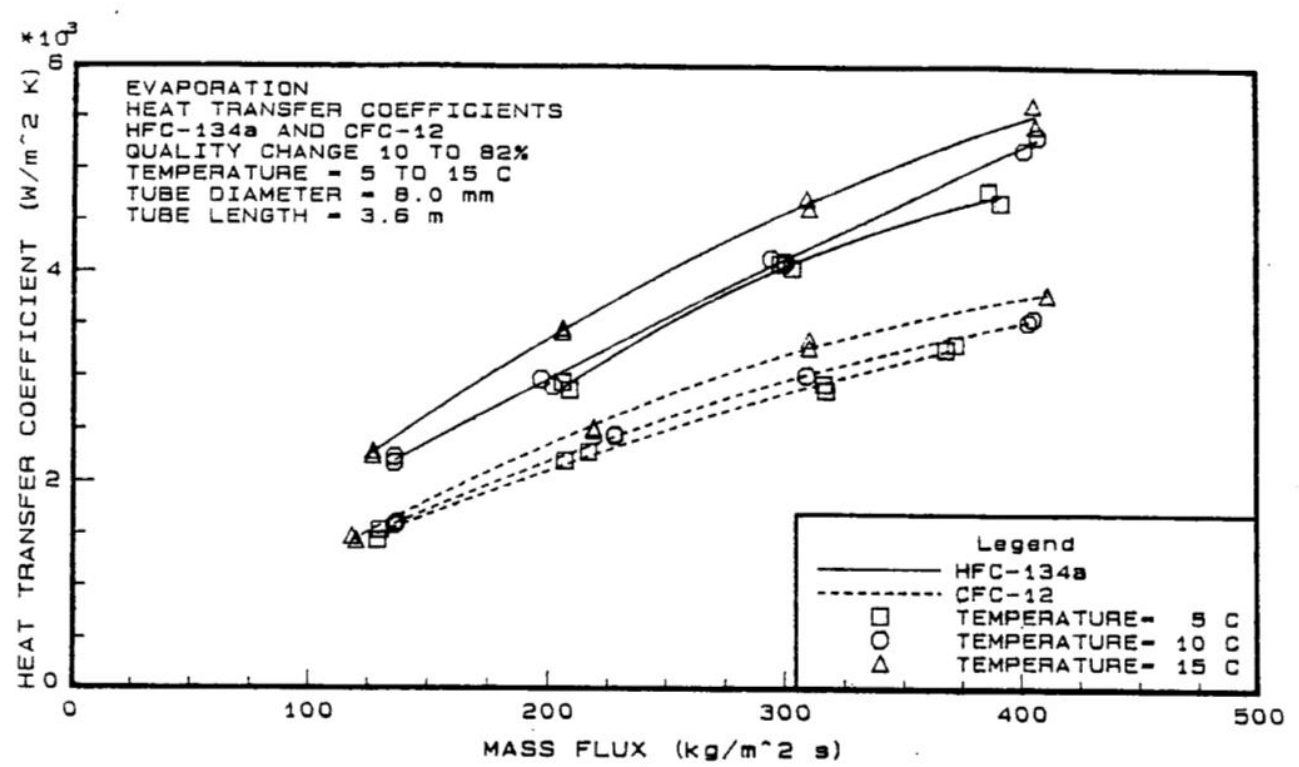

Figure 4 Measured evaporation heat transfer coefficients for HFC-134a and CFC-12 at three temperatures. 
Eckels, S.J. and M.B. Pate, An experimental comparison of evaporation and condensation heat transfer coefficients for HFC-134a and CFC-12. International Journal of Refrigeration, 1991. 14(2): p. 70-77.

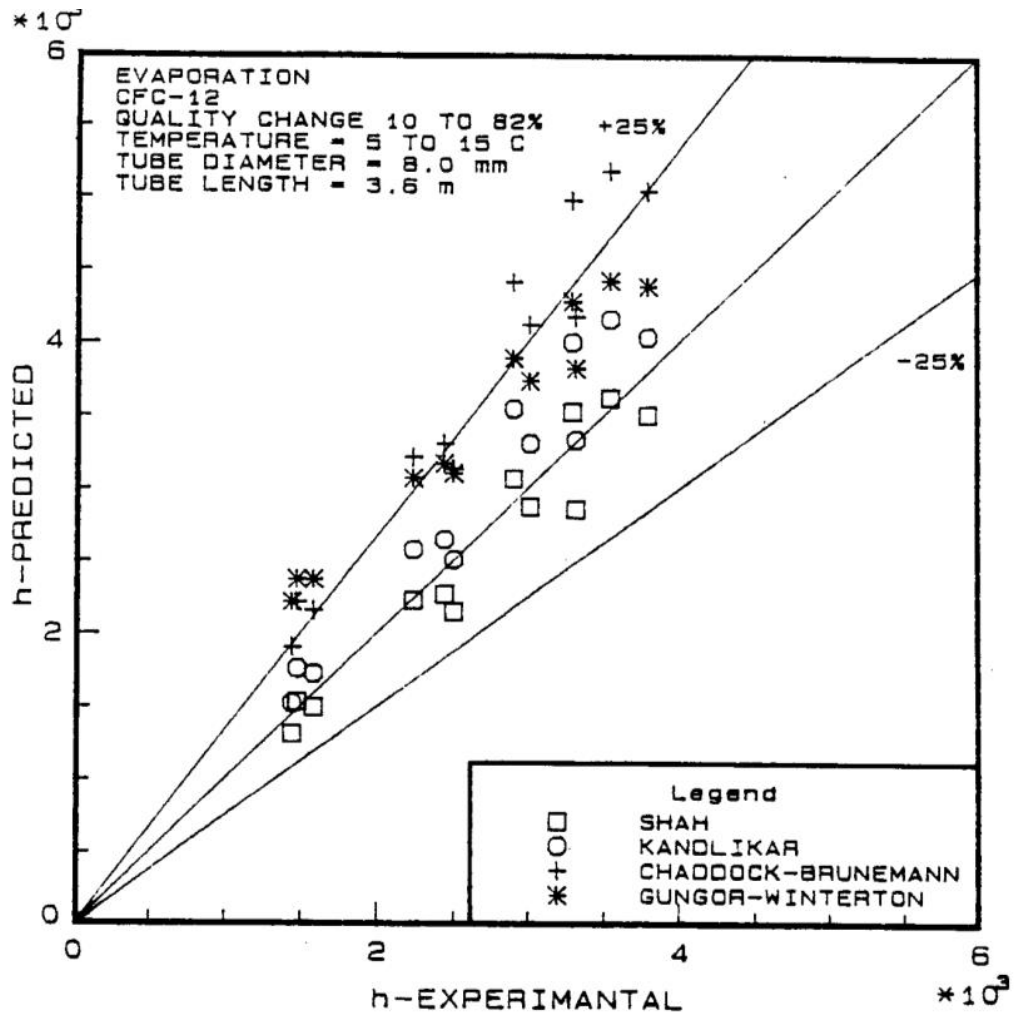

Figure 5 Predicted heat transfer coefficients for CFC-12 evaporation versus experimentally determined heat transfer coefficients.

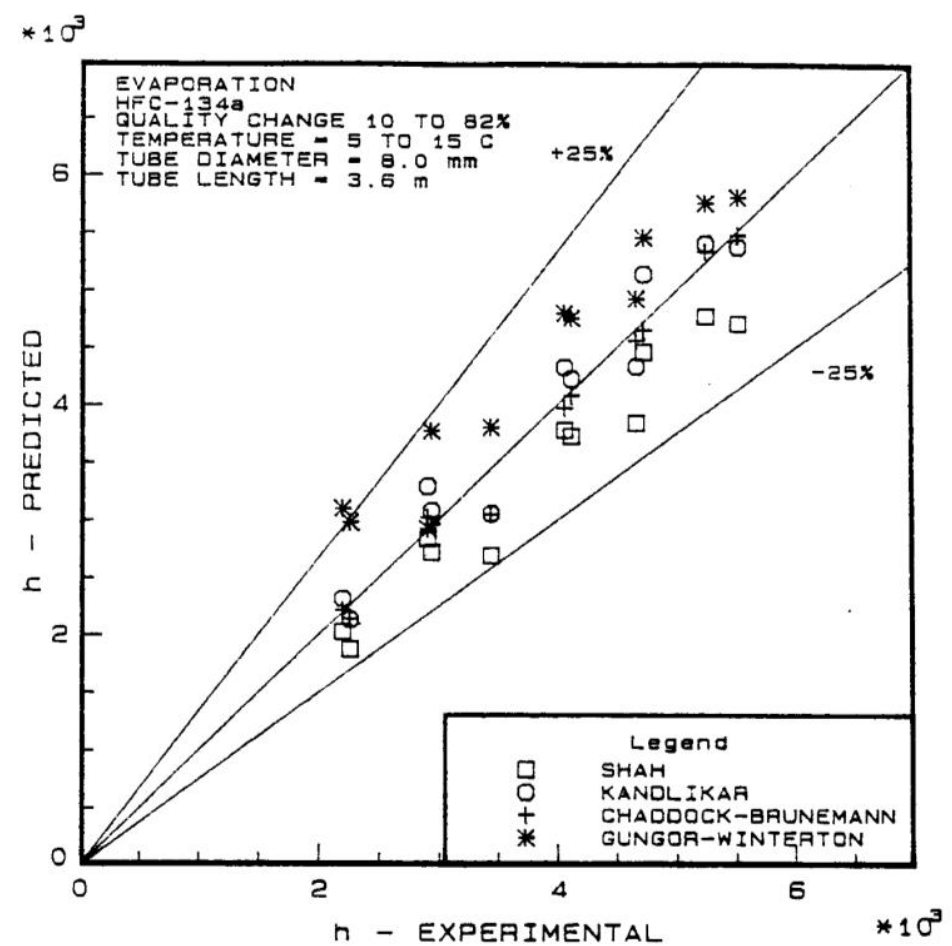

Figure 6 Predicted heat transfer coefficients for HFC-134a evaporation versus experimentally determined heat transfer coefficients. 
Eckels, S.J. and M.B. Pate, An experimental comparison of evaporation and condensation heat transfer coefficients for HFC-134a and CFC-12. International Journal of Refrigeration, 1991. 14(2): p. 70-77.

\section{Condensation}

Condensation tests were performed for a range of mass fluxes at three temperatures, 30,40 and $50^{\circ} \mathrm{C}$. As in the evaporation tests, variations of $\pm 1^{\circ} \mathrm{C}$ occurred at each temperature because of experimental limitations. The conditions for condensation tests are summarized in Table 2. As in the evaporation tests, the heat flux for HFC134a was higher than that for CFC-12 because of the increased enthalpy of vaporization. However, it should be noted that condensation heat transfer coefficients are not functions of heat flux.

Table 2 Condensation test conditions

\begin{tabular}{|l|l|l|}
\hline Parameter & CFC-12 & HFC-134a \\
\hline Temperature $\left({ }^{\circ} \mathrm{C}\right)$ & $30-50$ & $30-50$ \\
\hline Pressure $(\mathrm{MPa})$ & $0.75-\mathrm{I} .24$ & $0.76-1.32$ \\
\hline Mass flux $\left(\mathrm{kg} /\left(\mathrm{m}^{2} \mathrm{~s}\right)\right)$ & $125-400$ & $125-400$ \\
\hline Quality in $(\%)$ & $80-88$ & $80-88$ \\
\hline Quality out $(\%)$ & $8-15$ & $10-20$ \\
\hline
\end{tabular}

Figure 7 presents condensation heat transfer coefficient data versus mass flux for HFC-134a and CFC-12. The lines are a least-squares fit of the data at each temperature. For both refrigerants, the heat transfer coefficients decrease with temperature, but increase with mass flux. Comparing the two refrigerants, HFC-134a results in a 25-35\% higher heat transfer coefficient. A more detailed comparison of the two refrigerants for condensation is presented in the next section.

The experimental heat transfer coefficients are compared with the Shah[10], Traviss et al.[11] , and Cavallini and Zecchin[12] correlations. Similar to the evaporation case, these local heat transfer coefficients were integrated over the quality range to obtain average heat transfer coefficients. Figure 8 shows that differences between experimental and predicted heat transfer coefficients for CFC- 12 during condensation are less than $\pm 25 \%$. Figure 8 also shows that the correlations underpredict the heat transfer coefficients at the lower mass fluxes and overpredict at the higher mass fluxes. For condensation of HFC-134a, Figure 9 shows that differences between experimental and predicted heat transfer coefficients are less than $\pm 25 \%$. Again, the correlations tend to underpredict at the lower mass fluxes and overpredict the heat transfer coefficients at the higher mass fluxes.

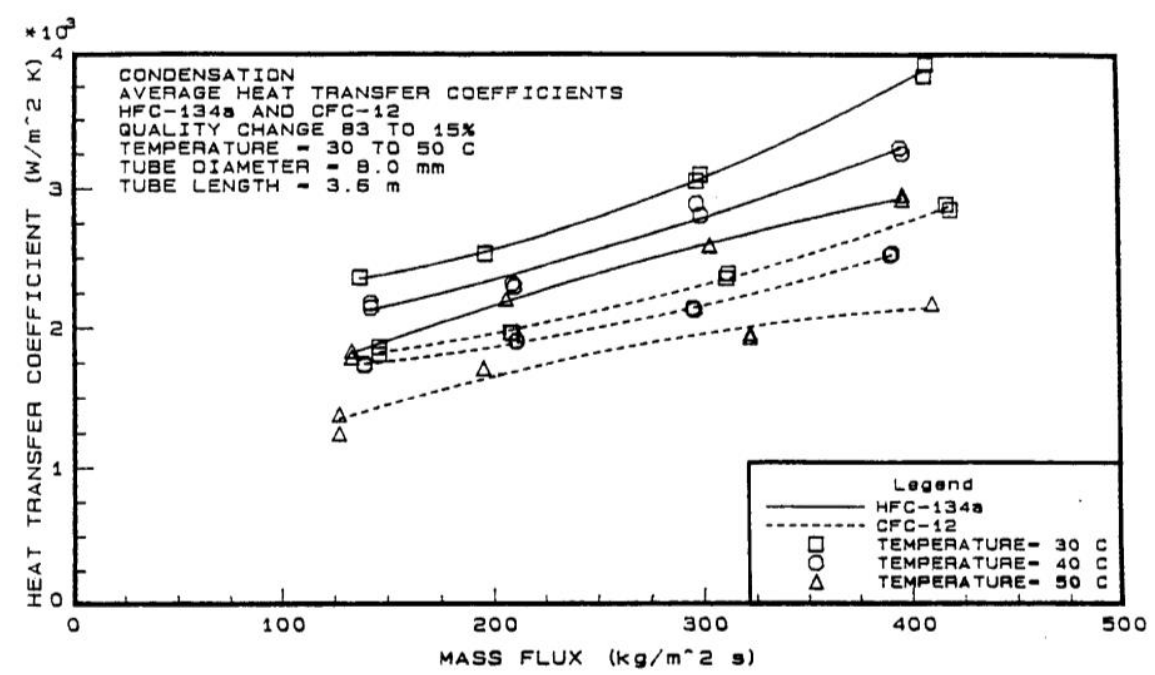

Figure 7 Measured condensation heat transfer coefficients for HFC-134a and CFC-12 at three temperatures. 
Eckels, S.J. and M.B. Pate, An experimental comparison of evaporation and condensation heat transfer coefficients for HFC-134a and CFC-12. International Journal of Refrigeration, 1991. 14(2): p. 70-77.

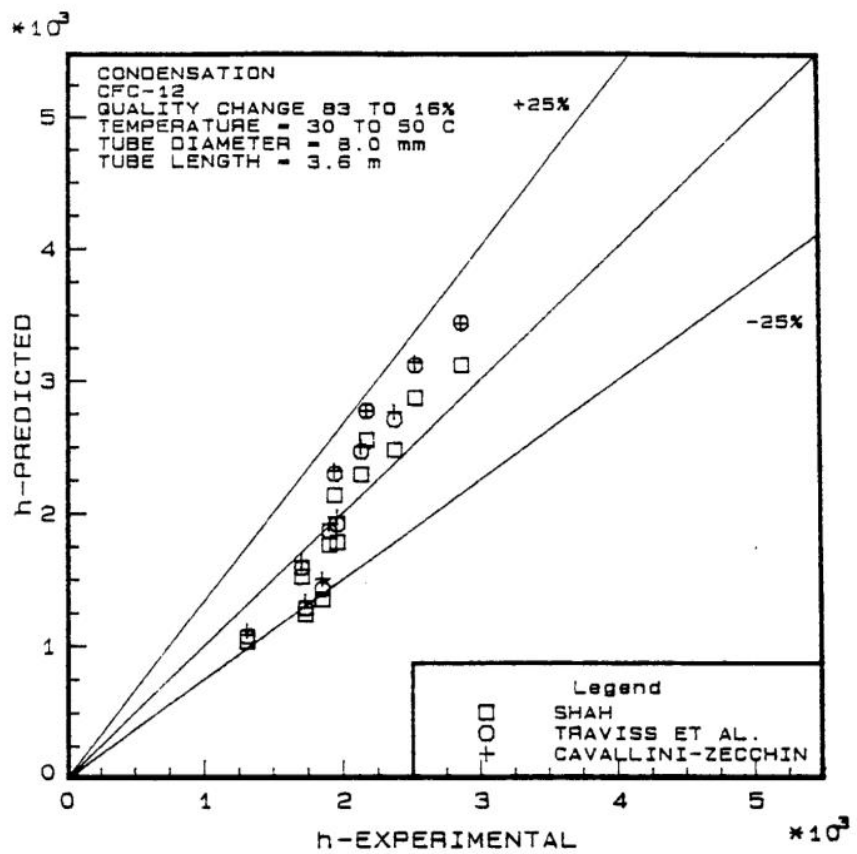

Figure 8 Predicted heat transfer coefficients for CFC-12 condensation versus experimentally determined heat transfer coefficients.

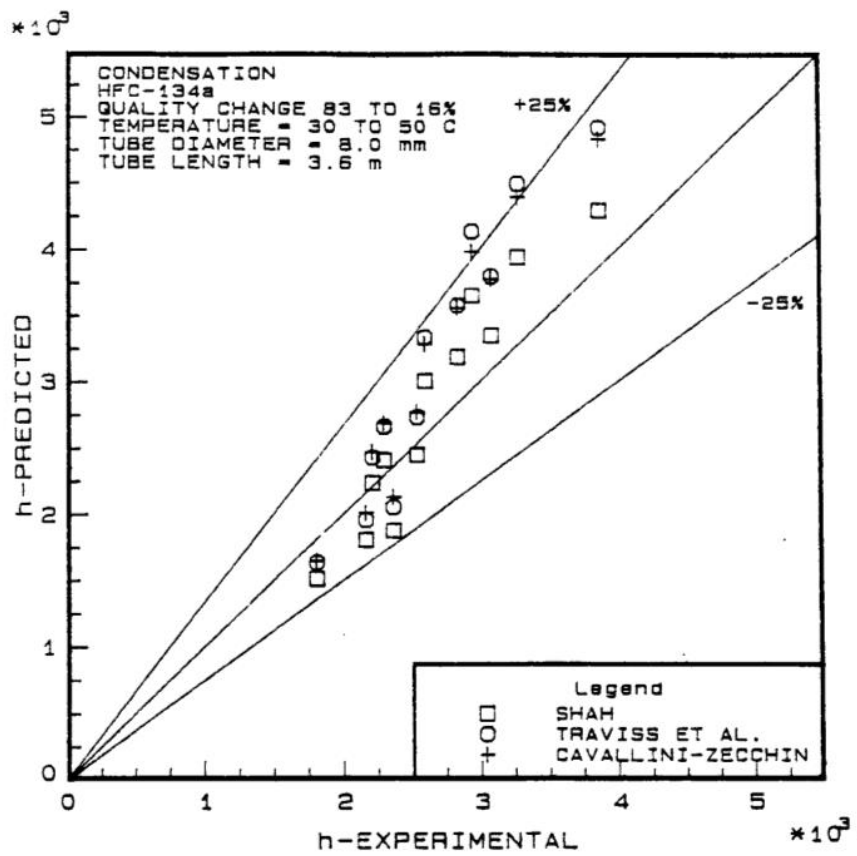

Figure 9 Predicted heat transfer coefficients for HFC-134 condensation versus experimentally determined heat transfer coefficients.

\section{Comparison of HFC-134a and CFC-12}

Modifying existing CFC-12 refrigeration components so that they perform at an optimum level with HFC-134a requires that the relationship between CFC-12 and HFC-134a heat transfer coefficients be quantified. Therefore, to aid the designer heat transfer coefficient ratios of the two refrigerants are presented. The heat transfer 
Eckels, S.J. and M.B. Pate, An experimental comparison of evaporation and condensation heat transfer coefficients for HFC-134a and CFC-12. International Journal of Refrigeration, 1991. 14(2): p. 70-77.

coefficients are compared on the basis of similar mass fluxes and similar cooling capacities. The cooling capacity comparison is based on approximating the cooling capacity by multiplying the mass flow-rate times the enthalpy of vaporization. For the condensation case, the value obtained is actually an equivalent heating rate.

\section{Equivalent mass fluxes}

Figure 10 shows the ratio ofHFC-134a to CFC-12 liquid single-phase heat transfer coefficients at the same mass flux. The ratio is formed from the HFC-134a and CFC-12 least-squares curve from Figure 2. The heat transfer coefficient ratio shown in Figure 10 varies from 1.31 to 1.33 over the mass flux range tested. Because of experimental constraints the ratio was not determined at different average temperatures for single-phase flow. Theoretically the ratio will change slightly with average temperature because of the changing ratio of thermal conductivity. For example at $-5^{\circ} \mathrm{C}$ the HFC-134a liquid thermal conductivity is $21.4 \%$ higher than for CFC-12 and at $40^{\circ} \mathrm{C}$ the HFC-134a liquid thermal conductivity is $16.9 \%$ higher than for CFC-12

Figure 11 compares the evaporation performance of the two refrigerants by presenting HFC-134a to CFC-12 heat transfer coefficient ratios. Again, this ratio was formed by using the least-squares fit of the heat transfer coefficients at each temperature range. Figure 11 shows that the ratio is 1.35 to 1.45 for evaporation. The ratio does not appear to vary systematically with temperature or mass flux. Part of the increase in heat transfer coefficients for HFC-134a is due to the increase in heat flux, as previously mentioned, but most of the increase is probaby due to the liquid thermal conductivity of HFC-134a, which is significantly higher than that of CFC-12.

Figure12 compares the condensation performance by using the same HFC-134a to CFC-12 heat transfer coefficient ratios. The ratio shows an increase of 1.25 to 1.35 for condensation of HFC-134a. Again, this ratio shows no real trend with temperature or mass flux. The increase in the condensation heat transfer coefficient for HFC-134a compared to CFC-12 is probably due to the higher liquid thermal conductivity of HFC-134a.

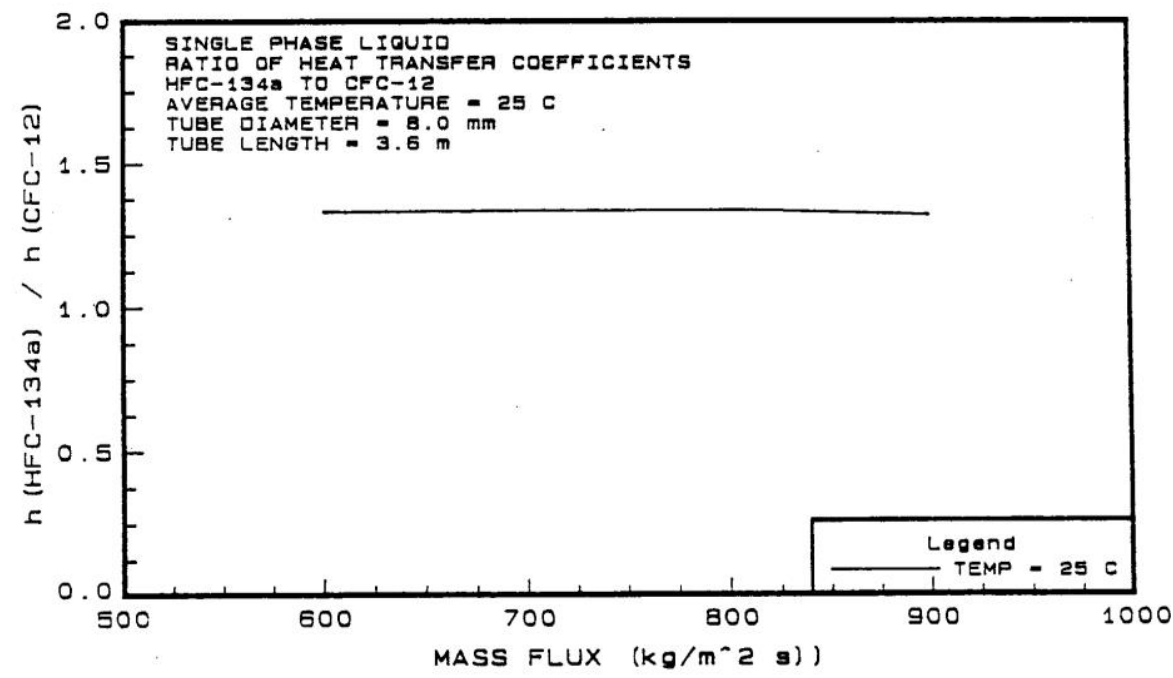

Figure 10 Ratio of HFC-134a to CFC-12 measured single- phase heat transfer coefficients; temperature $23^{\circ} \mathrm{C}$ 
Eckels, S.J. and M.B. Pate, An experimental comparison of evaporation and condensation heat transfer coefficients for HFC-134a and CFC-12. International Journal of Refrigeration, 1991. 14(2): p. 70-77.

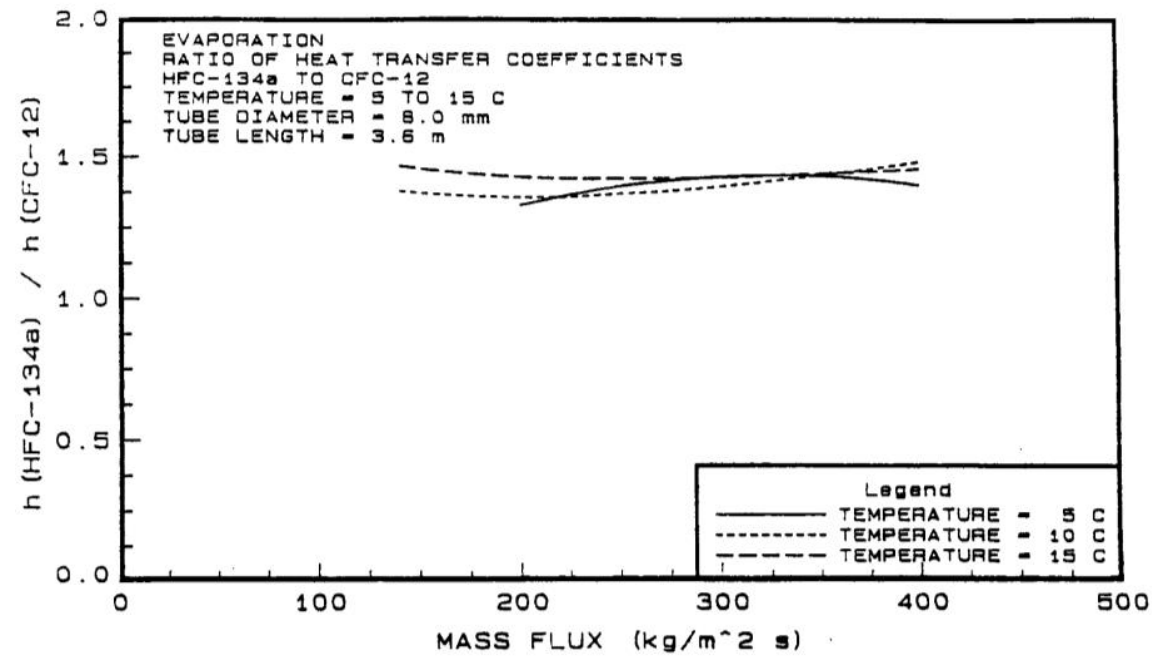

Figure 11 Ratio of HFC-134a to CFC-12 measured heat transfer coefficients for evaporation.

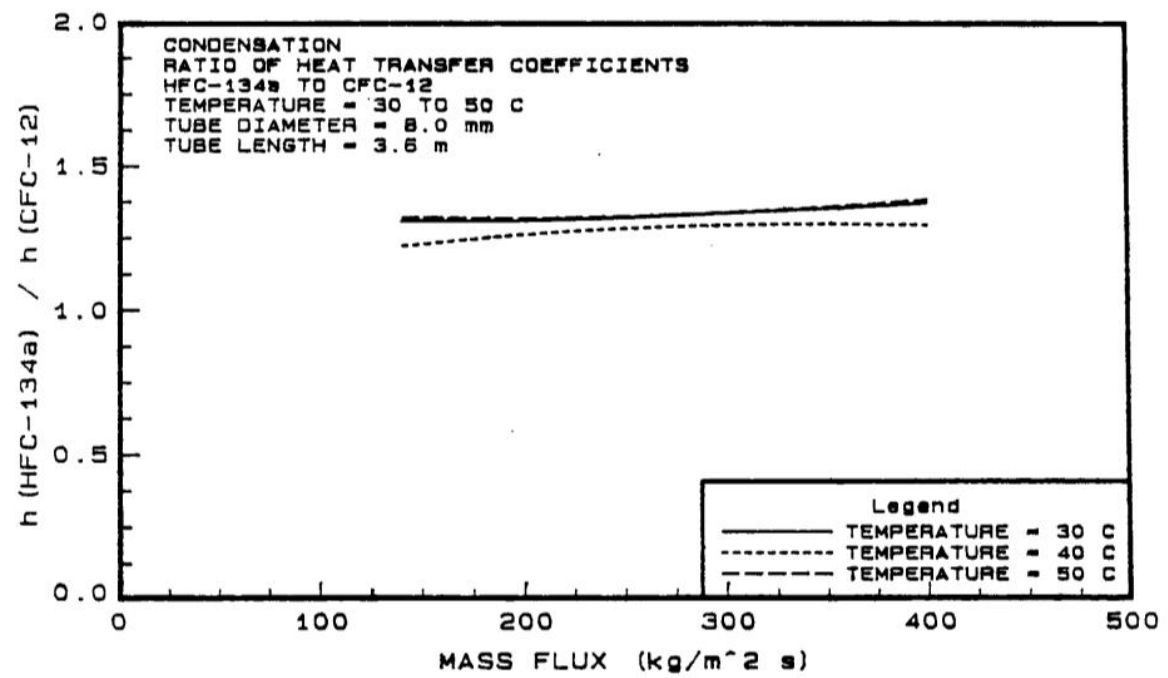

Figure 12 Ratio of HFC-134a to CFC-12 measured heat transfer coefficients for condensations.

Equivalent cooling (heating) capacity

Figure 13 compares the evaporation heat transfer coefficients for HFC-134a and CFC-12 at equivalent cooling capacity. The equivalent cooling capacity ratio is formed from the least-squares fit of the evaporation heat transfer coefficients found in Figure 4. Specifically, this ratio is formed from heat transfer coefficients taken at equivalent values of mass flow-rate times the enthalpy of vaporization of the refrigerant. As the enthalpy of vaporization is higher for HFC-134a, the heat transfer coefficient ratio is formed with the HFC-134a flowrate being significantly reduced compared to CFC-12. Figure 13 shows that even with the decreased mass flowrate, the HFC-134a to CFC-12 ratio is still 1.05 to significantly reduced compared to CFC-12. For this situation, HFC134a resulted in 5-15\% higher heat transfer coefficients. When the two refrigerants are compared for equivalent heating capacity in a condenser, HFC-134a resulted in heat transfer coefficients that were 10-20\% higher. 1.15, with the larger values occurring at the higher temperatures and cooling capacities. 
Eckels, S.J. and M.B. Pate, An experimental comparison of evaporation and condensation heat transfer coefficients for HFC-134a and CFC-12. International Journal of Refrigeration, 1991. 14(2): p. 70-77.

Figure 14 shows the HFC-134a to CFC-12 heat transfer coefficient ratio for condensation versus the equivalent heating capacity. The ratio is formed from the least-squares fit of the heat transfer coefficients in Figure 6.

Because of the increased enthalpy of vaporization for HFC-134a, the mass flowrate of HFC-134a was decreased by $15-20 \%$ to reflect equivalent heating capacity. The ratio of condensation heat transfer coefficients is 1.10 to 1.20 .

\section{Conclusions}

Heat transfer coefficients were experimentally determined for HFC-134a and CFC-12. In single-phase flow, heat transfer coefficients for HFC-134a were 33\% higher when compared to those of CFC-12. For evaporation at similar mass fluxes, HFC-134a heat transfer coefficients were 35 to $45 \%$ higher than those ofCFC- 12 . For condensation at similar mass fluxes, HFC-134a heat transfer coefficients were 25 to $35 \%$ higher than those of CFC-12. The two refrigerants were also compared for equivalent cooling capacity, obtained by multiplying mass flowrate and enthalpies of vaporization. As the enthalpy of vaporization is higher for HFC-134a, the heat transfer coefficient comparison is made with the HFC-134a flowrate being

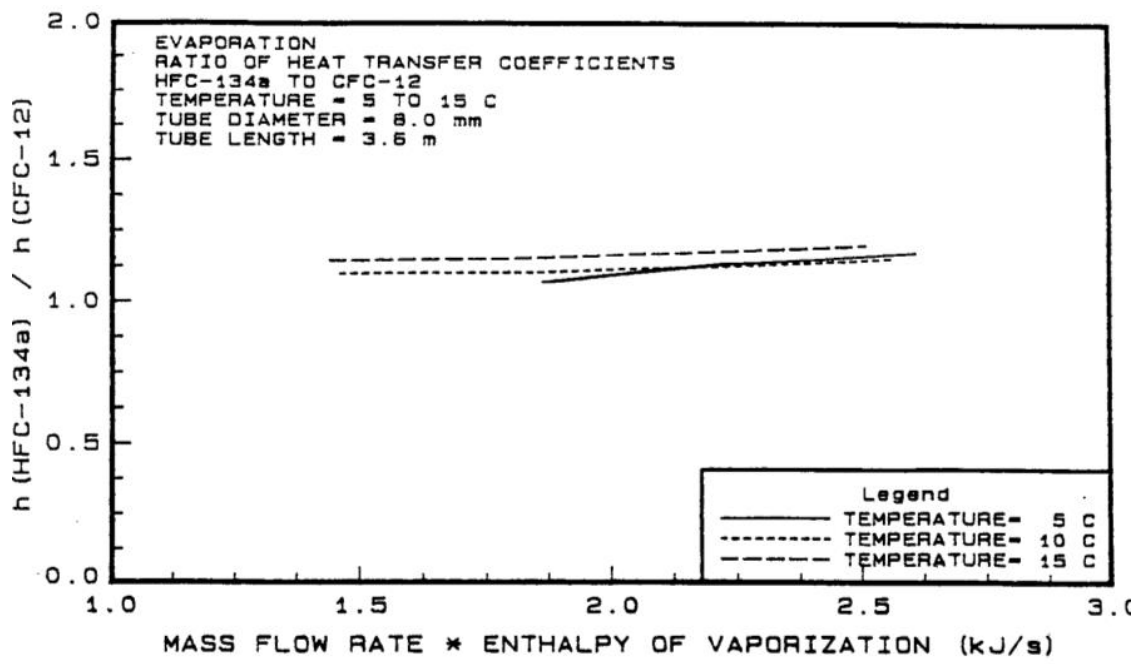

Figure 13 Ratio of HFC-134a to CFC-12 measured heat transfer coefficients for similar cooling capacity during evaporation.

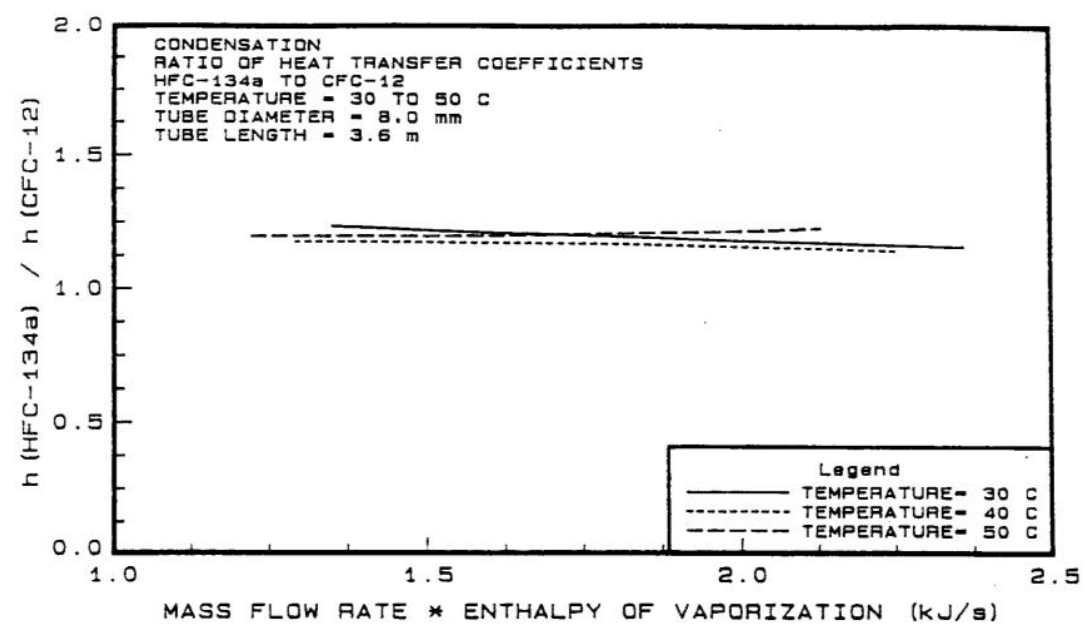

Figure 14 Ratio of HFC-134a to CFC-12 measured heat transfer coefficients for similar heating capacity during condensation. 
Eckels, S.J. and M.B. Pate, An experimental comparison of evaporation and condensation heat transfer coefficients for HFC-134a and CFC-12. International Journal of Refrigeration, 1991. 14(2): p. 70-77.

\section{Acknowledgements}

The authors thank E. I. Du Pont De Nemours \& Company (Inc.) for sponsoring this project and for supplying the HFC-134a. The authors also extend a special thanks to Dr Don Bivens of Du Pont for his support and helpful suggestions.

\section{References}

1. Schlager, L. M., Pate, M. B., Bergles, A. E. Evaporation and condensation of refrigerant-oil mixtures in a smooth tube and micro-fin tube ASHRAE Trans (1988) 93 293-416

2. Kline, S. J., McClintock, F. A. Describing uncertainties in simple sample experiments Meclz Eng (1953) 75 3-8

3. Bivens, D. B. E. I. Du Pont De Nemours \& Company, Inc. personal communication (1989)

4. McAdams, W. H. Heat Transmission (2nd ed.) McGraw-Hill, New York, USA (1942)

5. Petukhov, B. S. Heat transfer and friction in turbulent pipe flow with variable physical properties Advances in Heat Transfer Vol6 Academic Press, New York, USA (1970) 503-564

6. Shah, M. M. Chart correlation for saturated boiling heat transfer: equations and further study ASHRAE Trans (1982) 88 66-86

7. Kandlikar, S. S. A general correlation for saturated two-phase flow and boiling heat transfer inside horizontal and vertical tubes Boiling and Condensation in Heat Transfer Equipment ASME, New York, USA (1987) 85 9-19

8. Chaddock, J. B., Brunemann, H. Forced Convection Boiling of Refriqerants in Horizontal Tubes-Phase 3, HL-113, Duke University School of Engineering, Durham, NC, USA (1967)

9. Gungor, K. E., Winterton, R. H. S. A general correlation for flow boiling tubes and annuli Int J Heat Mass Flux (1986) 29 351-358

10. Shah, M. M. A general correlation for heat transfer during film condensation inside pipes $1 \mathrm{~m} J$ Heat Mass Transfer (1979) 88 185-196

11. Traviss, D. P., Rohsenow, W. M., Baron, A. B. Forced convection condensation inside tubes: A heat transfer equation for condenser design ASHRAE Trans (1972) 79 157-165

12. Cavallini, A., Zecchin, R. A dimensionless correlation for heat transfer in forced convection condensation In: Proceedings of the Fifth International Heal Transfer Conference Tokyo (1974) Vol3 309-313 